\title{
Correction to: Optical Interventions for Myopia Control
}

Wing Chung Tang, Myra Leung, Angel C. K. Wong,

Chi-ho To, and Carly S. Y. Lam

\section{Correction to: M. Ang, T. Y. Wong (eds.), Updates on Myopia,} https://doi.org/10.1007/978-981-13-8491-2_14

In the original version of Chapter 14, the chapter author name was wrongly given as Wing Chung Tang.

The name of the author should be Wing Chun Tang.

The updated online version of this chapter can be found at https://doi.org/ 10.1007/978-981-13-8491-2_14. 